\title{
非贵金属催化酮的不对称硅氢加成反应的研究进展
}

\author{
刘帅彭家建*厉嘉云白赢肖文军来国桥* \\ (杭州师范大学有机硅化学及材料技术教育部重点实验室 杭州 310012)
}

\begin{abstract}
摘要 手性二级醇作为手性药物、香料等的原料，主要通过不对称加氢来制备. 近来通过不对称硅氢加成的方法来制 备手性醇的方法，由于其温和的反应条件及相对低廉的催化剂成本而受到广泛关注. 介绍了近年来锌、铁、铜、钛等 非贵金属催化剂催化潜手性酮的不对称硅氢加成反应的研究进展.
\end{abstract}

关键词 不对称硅氢加成; 非贵金属催化剂; 手性醇; 潜手性酮; 进展

\section{Progress in Asymmetric Hydrosilylation of Ketones by Non-precious Metal Catalyst}

\author{
Liu, Shuai Peng, Jiajian* Li, Jiayun Bai, Ying Xiao, Wenjun Lai, Guoqiao* \\ (Key Laboratory of Organosilicon Chemistry and Material Technology, Ministry of Education, Hangzhou Normal University, \\ Hangzhou 310012)
}

\begin{abstract}
As important intermediates for the development of biologically active molecules and fragrance etc., chiral secondary alcohols are synthesized through asymmetric hydrogenation. Recently, the asymmetric hydrosilylation process has been attracted because of its mild reaction conditions as well as inexpensive and safer reagent system. In this paper, some information about the recent development of the catalysts of the non-precious metal ( $\mathrm{Zn}, \mathrm{Cu}$ and so on) for asymmetric hydrosilylation reaction of prochiral ketones is briefly reviewed.
\end{abstract}

Keywords asymmetric hydrosilylation; non-precious metal catalyst; chiral alcohol; prochiral ketone; progress

手性二级醇在工业上具有广泛的用途，是合成手性 药物、农业化学品、香料和液晶等物质的重要中间 体 ${ }^{[1 \sim 4]}$. 手性二级醇通常经由潜手性酮不对称加氢 ${ }^{[5]}$ 、氢 转移氢化 ${ }^{[6]}$ 、手性硼杂噁唑烷催化还原 ${ }^{[7]}$ 等方法来制备. 其中不对称加氢、氢转移氢化两种方法均涉及手性过渡 金属催化剂的研究, 而潜手性酮与手性硼试剂加成再水 解制备手性二级醇的过程主要涉及手性氨基醇衍生的 手性嗍试剂研究. 潜手性酮不对称硅氢化是不对称加氢 的重要方法之一, 与使用氢气相比, 使用氢硅烷作为氢 源, 其主要的优点是条件特别的温和, 无需耐高压技术, 可操作性强. 通过催化潜手性酮的不对称硅氢加成反 应，可以简单、高效地制备得到手性二级醇，如图 1 .

经过多年研究和探索, 在潜手性酮的不对称催化方 面, 人们已经研发出了多种不对称硅氢加成反应金属催 化剂, 1973 年 Kagan 等 ${ }^{[8,9]}$ 首次报道 Rh(I)/DIOP 催化潜

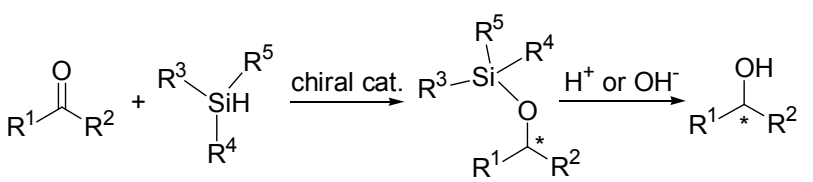

图 1 酩的不对称硅氢加成反应

Figure 1 Asymmetric hydrosilylation of ketones

手性酮和亚胺不对称硅氢加成. 在这之后, 大量的研究 集中在高效催化剂的制备上. 早期的不对称硅氢加成反 应催化剂主要局限于使用 $\mathrm{Rh}, \mathrm{Ir}$ 和 $\mathrm{Ru}$ 等贵金属, 存在着 催化剂成本较高, 难以重复利用, 以及催化剂流失等问 题 ${ }^{[10 ~ 14]} .20$ 世纪 90 年代, 随着各种非贵金属催化剂如 $\mathrm{Zn}, \mathrm{Cu}, \mathrm{Fe}$ 等新型催化剂对不对称硅氢加成反应催化性 能的深入研究, 发现非贵重金属催化剂在特定条件下对 酮的硅氢加成反应也具有高活性和立体选择性. 特别是 新颖的价格低廉的铜、锌的催化体系的发展，大大拓宽

\footnotetext{
*E-mail: jjpeng@hznu.edu.cn; gqlai@hznu.edu.cn

Received February 7, 2012; revised April 11, 2012; published online May 21, 2012.

Project supported by the Aid Program for Key Science and Technology Innovative Research Team in Zhejiang Province (No. 2009R50016) and the Natural Science Fundation of Zhejiang Province (No. Y4100248).

浙江省重点科技创新团队专项(No. 2009R50016)、浙江省自然科学基金(No. Y4100248)资助项目.
} 
了非贵金属催化剂的应用前景. 本文重点介绍近年来 锌、铁、铜、钛等非贵金属催化剂催化潜手性酮的不对 称硅氢加成反应的研究进展.

\section{1 潜手性酮不对称硅氢加成反应中的锌催化剂}

1999 年, Mimoun 等 ${ }^{[15]}$ 制备了手性二胺 $(\mathbf{1 a} \sim \mathbf{1 g})$, 将其作为二乙基锌的手性配体, 催化潜手性酮的不对称 硅氢加成反应, 如图 2. 结果表明: 在各种催化体系中 由手性的二胺配体与二乙基锌的络合物对催化潜手性 酮的不对称硅氢加成反应非常有效, 配体 1f (ebpe)-二 乙基锌催化体系的立体选择性最好, 催化苯乙酮的不对 称硅氢加成不对称选择性可达到 $88 \%$ ee.

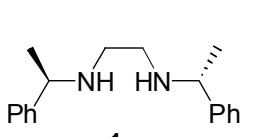

$1 \mathrm{a}$

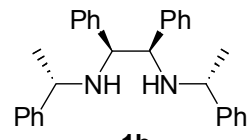

$1 b$

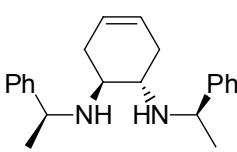

$1 d$

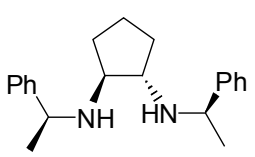

$1 e$
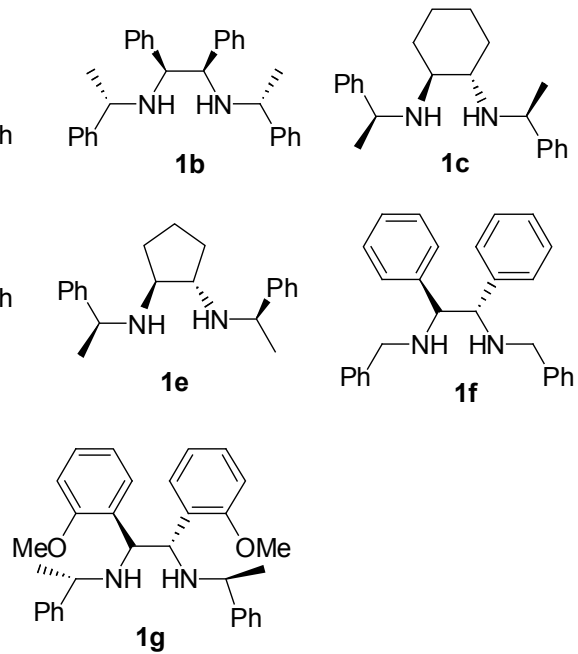

图 2 手性二胺配体的结构

Figure 2 Structures of chiral diamine ligands

Bette 课题组 ${ }^{[16,17]}$ 对二烷基锌-二胺金属催化体系催 化潜手性酮的不对称硅氢加成反应做了大量的研究. 研 究了不同的二烷基锌-二胺催化体系催化苯乙酮的不对 称硅氢加成反应, 主要讨论了配体的组成和立体结构对 催化活性和立体选择性的影响. 研究者制备了一系列的 手性二胺配体, 考察了不同配体和二烷基锌催化体系对 反应的影响 ${ }^{[18,19]}$. 研究表明, 选用配体 1c 与二乙基锌配 位催化苯乙酮的立体选择性高达到 $91 \%$, 但反应速度非 常缓慢, 反应 $228 \mathrm{~h}$ 产率才只有 66\%. 2005 年, Mikami 课题组 ${ }^{[20]}$ 利用二乙基锌-二胺 $(\mathbf{2} \mathbf{a} \sim \mathbf{2 d})$ 催化酮的硅氢加 成反应，如图 3. 当 $\mathrm{X}=\mathrm{F}$ 时, $10 \mathrm{~mol} \%$ 二乙基锌/二胺催 化剂, 2.5 equiv. 含氢硅烷, 催化 2,4,4',6-四甲基二苯甲酮 时光学选择性为 $96 \%$, 转化率高达 $97 \%$.

Mimoun 等 ${ }^{[15]}$ 根据实验数据和模型推理提出了 $\mathrm{Zn} /$ 手性二胺催化酮的不对称硅氢加成的反应机理, 如图 4 所示, 首先酩插入到 $\mathrm{Zn}-\mathrm{N}$ 中, 形成一个七元环中间体 b, 中间体再与氢硅烷生成 $\mathrm{Zn}-\mathrm{H}(\mathbf{c})$, 转移到潜手性酮
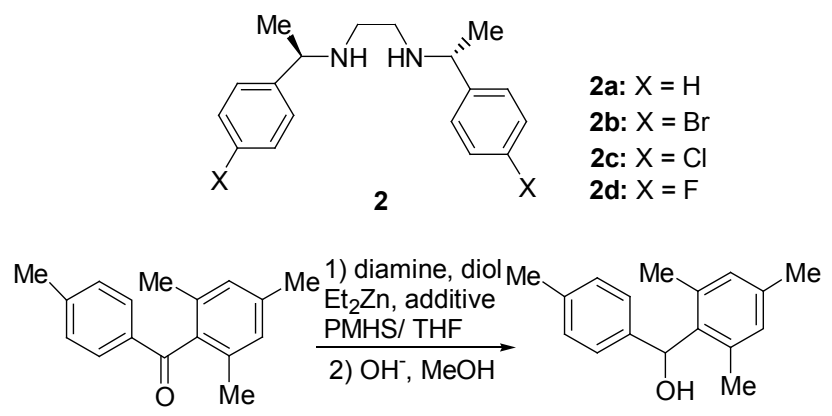

图 3 二乙基锌-二胺催化酮的不对称硅氢加成反应

Figure 3 Asymmetric hydrosilylation of ketone catalyzed by diethylzinc and diamine ligands

上, 释放出稳定的硅醚, 同时 $\mathrm{Zn}$ 的络合物与下一分子 酮形成中间体 $\mathbf{a}$, 从而进入下一轮催化.

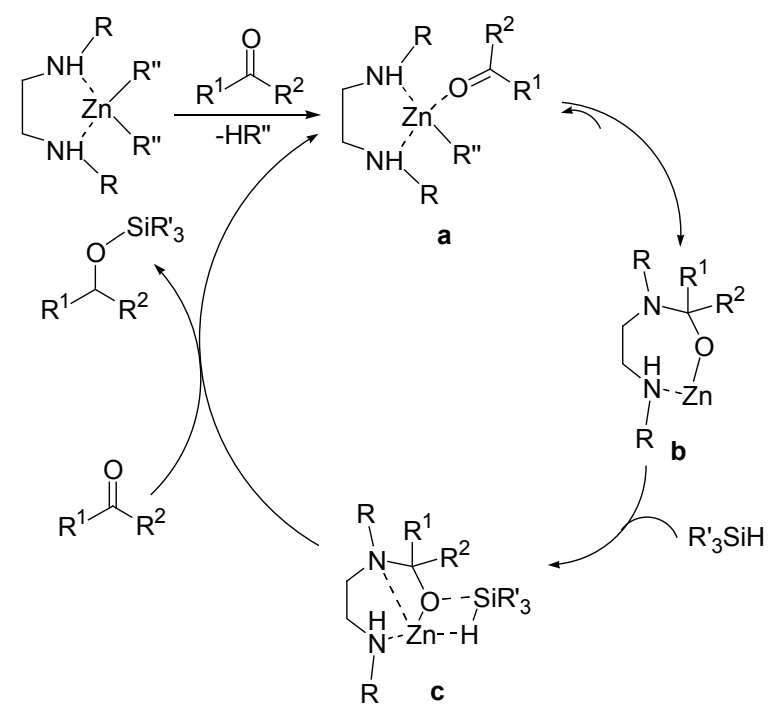

图 $4 \mathrm{Zn}$ /手性二胺催化潜手性酮的不对称硅氢加成反应机理 Figure 4 Mechanism of hydrosilylation of ketones catalyzed with $\mathrm{ZnEt}_{2} /$ chiral diamine

2008 年, Nishiyama 课题组 ${ }^{[21]}$ 发现手性 $\mathrm{N}_{2} \mathrm{~S}_{2}$ 配体 $3 \mathbf{a} \sim \mathbf{4 b}$ 与醋酸锌配位，以 $(\mathrm{EtO})_{2} \mathrm{MeSiH}$ 为氢源，能直接 高效地催化一系列芳基酮的不对称硅氢加成反应，其中 以对 $\alpha$-䒬乙酮的催化效果最好，光学选择性高达 $92 \%$, 产率达到 $95 \%$, 但对其它的芳基酮的不对称选择性有一 定的局限性.
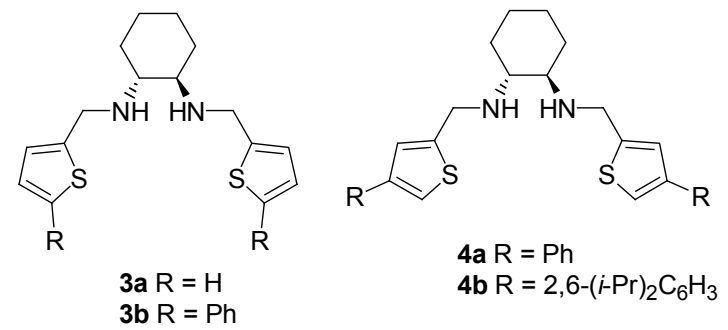

图 5 手性 $\mathrm{N}_{2} \mathrm{~S}_{2}$ 配体

Figure 5 Chiral $\mathrm{N}_{2} \mathrm{~S}_{2}$ ligands 
2009 年, Gajewy 等 ${ }^{[22]}$ 再次利用二乙基锌和环状配 体 $\mathbf{5 a} \sim \mathbf{5 f}$, 催化酮的硅氢加成反应, 如图 6. 研究发现, 当使用 $5 \mathbf{a}$ 配体时, 催化体系没有活性; 而使用 $\mathbf{5 b}$ 配体 时, 以甲苯为溶剂, 用一苯基含氢硅烷作为氢源, 转化 率最高达到 $99 \%$, 但光学选择性只有 58\%, 用二苯基含 氢硅烷作为氢源时, 光学选择性为 $89 \%$, 转化率为 $85 \%$; 使用配体 $\mathbf{5 c}$ 时, 同样以二苯基含氢硅烷作为氢源, 光学 选择性最高为 $82 \%$, 转化率高达 $99 \%$; 使用配体 $5 \mathbf{d} \sim 5 \mathbf{5 f}$ 时, 相对来说转化率仍然在 $90 \%$ 以上, 但是光学选择性 则比较低.

2012 年, Beller 课题组 ${ }^{[23]}$ 合成了一系列双啞唑啉或 咪唑类配体 6a 7c, 如图 7 所示, 并主要考察了它们与
二乙基锌或醋酸锌原位催化潜手性酮的不对称硅氢加 成反应, 研究发现这类配体除 7c 外都能与二乙基锌或 醋酸锌高效地催化酮的硅氢加成反应. 室温条件下，以 5 equiv. PMHS 为氢源, THF 为溶剂, 用二乙基锌与配体 6a 7 $\mathbf{b}$ 原位催化苯乙酮的不对称硅氢加成, 产率都> $95 \%$, 但遗憾的是不对称选择性最高只有 $59 \%$. 该催化 体系对不同的酮的底物有着普遍的适用性，室温下，以 配体 6a 与二乙基锌原位催化联苯乙酮与 PMHS 的硅氢 加成反应, 产率可达到 $99 \%$, 不对称选择性可以达到 $73 \%$. 同时, Beller 课题组还通过 ESI-TOF-MS 技术检测 到反应中的活化物种, 进一步验证了 Mimoun 提出的反 应机理.

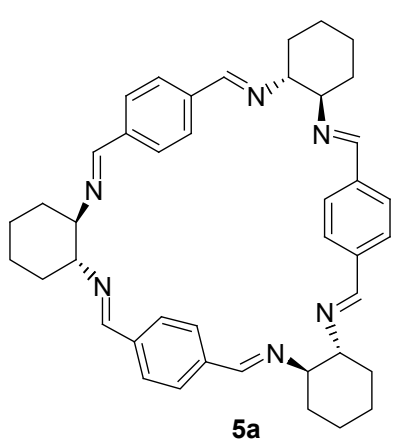

$5 a$

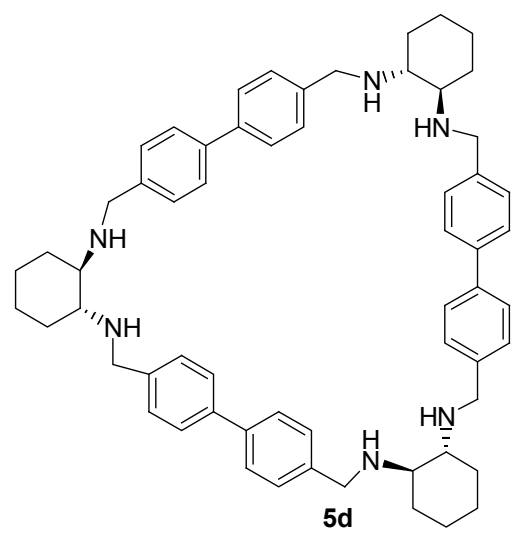

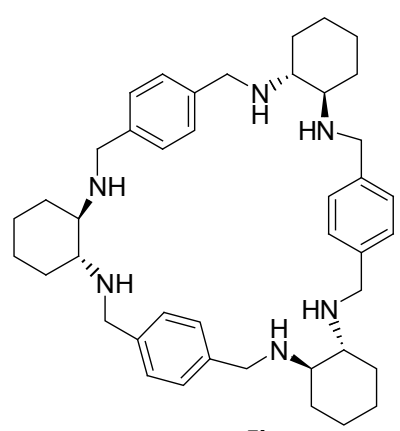

$5 b$

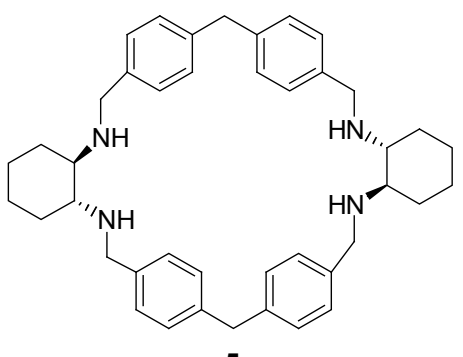

$5 e$

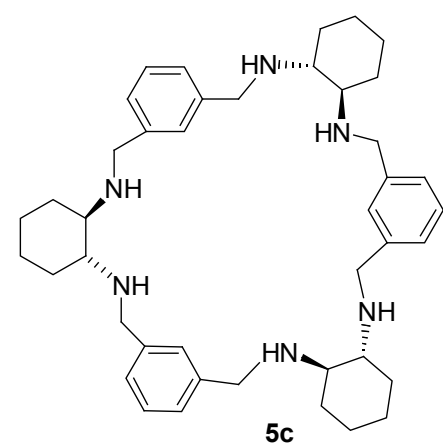

5c<smiles>c1ccc(CNC2CCCCC2NCc2ccccc2)cc1</smiles>

$5 f$

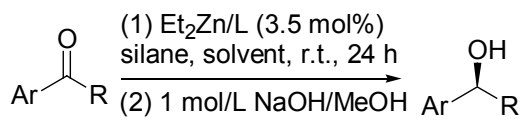

图 6 二乙基锌以及环状配体催化酮硅氢加成反应

Figure 6 Asymmetric hydrosilylation of ketone catalyzed with diethylzinc and macrocyclic trianglamine ligand<smiles>[R]C1COC(c2cccc(C3=NC([R])CO3)n2)=N1</smiles>

6a: $\mathrm{R}=(R)-\mathrm{Ph}$

6b: $\mathrm{R}=(S)-i-\mathrm{Pr}$

6c: $\mathrm{R}=(S)-\mathrm{PhCH}_{2}$<smiles>c1cc(C2=NC3c4ccccc4CC3O2)nc(C2=NC3c4ccccc4CC3O2)c1</smiles>

6d<smiles>[3H]N1C(c2cccc(C3=NC(c4ccccc4)C(c4ccccc4)N3[AlH2-])n2)=NC(c2ccccc2)C1c1ccccc1</smiles><smiles>[R]N1C(c2cccc(C3=N[C@@H](c4ccccc4)C(c4ccccc4)N3[R])n2)=N[C@@H](c2ccccc2)C1c1ccccc1</smiles>

$\mathrm{R}=p-\mathrm{MeOC}_{6} \mathrm{H}_{4} \mathrm{C}(\mathrm{O})$

图 7 不同的吡啶类化合物衍生的配体 $6 \mathbf{a} \sim 7 \mathbf{c}$

Figure 7 Screening of different pybox $\mathbf{6 a} \sim \mathbf{6 e}$ and pybim ligands $\mathbf{7 a} \sim \mathbf{7 c}$ 
Liu 等 ${ }^{[24]}$ 合成了一系列 $\alpha$-氨基酸水杨醛席夫碱配体 8a $\sim 8 f$, 如图 8 所示, 并用其与二乙基锌原位催化潜手 性酮不对称硅氢加成反应, 发现这类催化剂有很高的催 化活性和不对映选择性, 尤其是当使用 $L$-缅氨酸与 3,5二叔丁基水杨醛合成的席夫碱 8a 作为配体, 与二乙基 锌原位催化苯乙酮的不对称硅氢加成反应时, 不对映选 择性可以达到 $96 \%$, 这是目前锌催化体系催化苯乙酮的 不对称硅氢加成反应中最高的光学选择性, 同时产率也 达到了 $71 \%$. 探究了反应温度和溶剂对催化反应的影 响，同时发现这类催化剂对芳基酮有着普遍的适用性. 在前人的研究和实验数据基础上提出了比较合理的催 化机理, 认为 $\mathrm{COOK}$ 在反应中扮演了重要的作用.<smiles>[R]C(=O)N=Cc1cc([13CH3])cc([13CH3])c1O</smiles>

\begin{abstract}
8a: $(R) \mathrm{R}=i-\operatorname{Pr}, \mathbf{8 b}:(S) \mathrm{R}=i-\operatorname{Pr}, \mathbf{8 c}:(R) \mathrm{R}=\mathrm{Me}$, 8d: $(R) \mathrm{R}=i-\mathrm{Bu}, \mathbf{8 e}:(S) \mathrm{R}=i-\mathrm{Bu}, \mathbf{8 f}:(R) \mathrm{R}=\mathrm{s}-\mathrm{Bu}$ 8g: $(R) \mathrm{R}=\mathrm{CH}_{2} \mathrm{Ph}$
\end{abstract}

图 8 席夫碱配体的结构

Figure 8 Structures of Schiff base ligands

\section{2 潜手性酮不对称硅氢加成反应中的铁催化剂}

早在 1990年, Brunner 等 ${ }^{[25]}$ 最先报道了 $[\mathrm{Fe}(\mathrm{Cp})(\mathrm{CO})]$ 络合物在光诱导下不对称催化苯乙酮的硅氢加成反应 催化性能. 而后, Nishiyama和 Furuta等 ${ }^{[26-28]}$ 对醋酸亚铁 与手性三齿的噁唑啉的配合物不对称催化潜手性酮硅 氢加成作了深入的研究, 如图 9 所示, 配体 9 的的不对 称选择性为 37\%, 配体 10a 和 10b 的不对称选择性分别 达到 57\%和 79\%, Nishiyama 课题组进一步研究发现噁 唑啉上的取代基位阻越大得到的不对称选择性越高, 当 使用配体 10c 时不对称选择性达到 $88 \%$.

最近 Nishiyama 课题组 ${ }^{[29]}$ 又报道了新型手性铁配合 物 11, 如图 10 所示, 以 1.5 equiv.二乙氧基甲基含氢硅 烷为氢源, 以正己烷为溶剂, 在 $50{ }^{\circ} \mathrm{C}$ 下, 催化 4-苯基苯 乙酮, 得到手性二级醇, 产率高达 $99 \%$, 但光学选择性 只有 $66 \%$.

Beller 课题组 ${ }^{[30]}$ 利用醋酸亚铁和配体 12a $\sim 12 \mathbf{h}$, 如 图 11, 以四氢呋喃为溶剂, 反应温度为 $65{ }^{\circ} \mathrm{C}$, 催化苯乙 酮与二乙氧基甲基含氢硅烷的硅氢加成反应, 光学选择 性最高达到了 $68 \%$, 以 $2,4,6$-三甲基苯乙酮为底物时, 光学选择性可高达 $99 \%$.

Chirik 课题组 ${ }^{[1]}$ 对铁催化不对称硅氢加成主要集中 在烃基铁与手性双噁唑啉的络合物合成与研究. 利用 $0.3 \mathrm{~mol} \%$ 烃基铁配合物(13a $\sim 13 \mathrm{c}$, 如图 12 所示)为催化 剂, $1 \mathrm{~mol}$ 苯乙酮以及 $2 \mathrm{~mol}$ 苯基含氢硅烷，以乙二醇二<smiles>[R]c1ccc(C(C)=O)cc1</smiles><smiles>[R]c1ccc([C@@H](C)O)cc1</smiles>

$\mathrm{R}=\mathrm{Ph}, \mathrm{Me}_{2} \mathrm{~N}$, Morph<smiles></smiles>

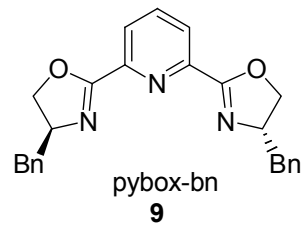

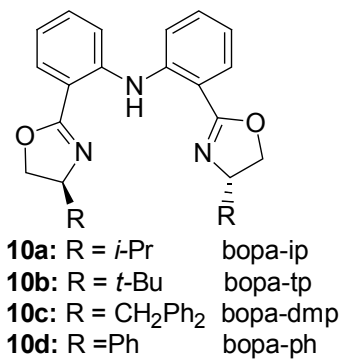

图 9 醋酸亚铁与手性噁唑啉类的化合物催化酮的不对称硅 氢加成反应

Figure 9 Asymmetric hydrosilylation of ketone catalyzed with $\mathrm{Fe}(\mathrm{OAc})_{2}$

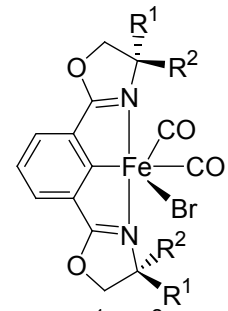

11a: $R^{1}=R^{2}=M e$

11b: $\mathrm{R}^{1}=i-\mathrm{Pr}, \mathrm{R}^{2}=\mathrm{H}$

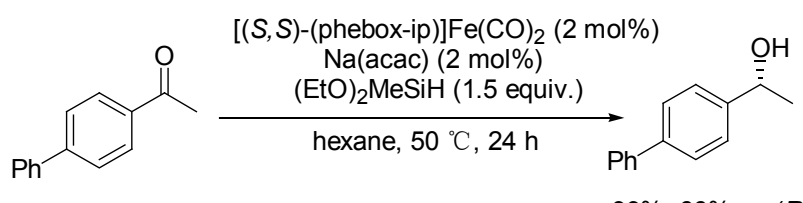

$99 \%, 66 \%$ ee $(R)$

图 10 手性铁配物 11 催化芳基酮的不对称硅氢加成反应

Figure 10 Asymmetric hydrosilylation of aromatic ketone catalyzed with a chiral iron complex $\mathbf{1 1}$

乙醚为溶剂, 室温下反应 $1 \mathrm{~h}$, 转化率最高可达到 $99 \%$, 光学选择性比较低, 最高只有 $49 \%$. 但 $\mathbf{1 0 a}$ 与 $\mathrm{B}\left(\mathrm{C}_{6} \mathrm{~F}_{5}\right)_{3}$ 一起使用, 其光学选择性可达到 $93 \%$.

Gade 课题组 ${ }^{[2]}$ 合成了一系列钳状的新型手性配体 14a 14i, 如图 13 所示, 并应用于酮的不对称硅氢加成 反应. 研究发现, 配体与醋酸铁能高效的催化各种酮的 不对称硅氢加成反应，其中以 $14 \mathrm{e}$ 与醋酸铁的络合物的 催化效果最好, 催化苯乙酮产率最高达到 $85 \%$, 不对称 选择性达到 86\%; 催化 2,4,6-三甲基苯乙酮，不对称选 择性可达到 93\%, 表现出优异的催化性能.

\section{3 潜手性酮不对称硅氢加成反应中的铜催化剂}

1984 年, Brunner 等 ${ }^{[33]}$ 首次将 $\mathrm{Cu}(\mathrm{I})$ 应用于催化 


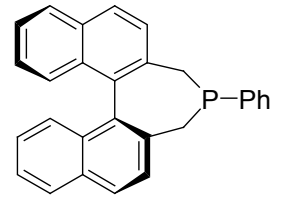

12a $(97 \%, 0 \%$ ee $)$

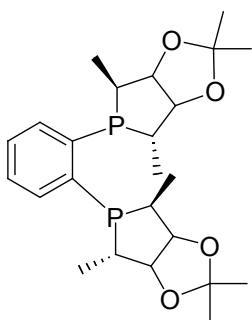

12e $(98 \%, 47 \%$ ee $)$

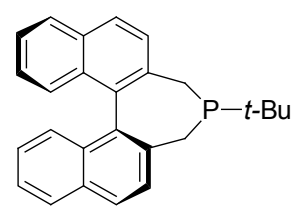

12b (>99\%, 0\% ee $)$
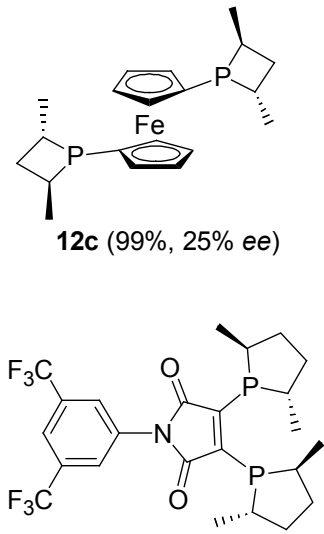

12g $(57 \%, 1 \%$ ee $)$

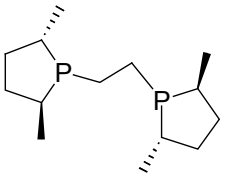

12d (15\%, 40\% ee)

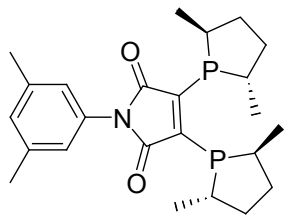

$12 \mathrm{f}(21 \%, 9 \%$ ee $)$

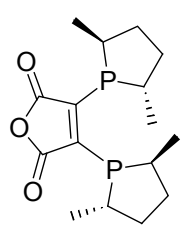

2h $(71 \%, 5 \%$ ee $)$

图 11 手性膦配体 $12 \mathrm{a} \sim 12 \mathrm{~h}$

Figure 11 Chiral phosphine ligands $12 \mathrm{a} \sim \mathbf{1 2 h}$
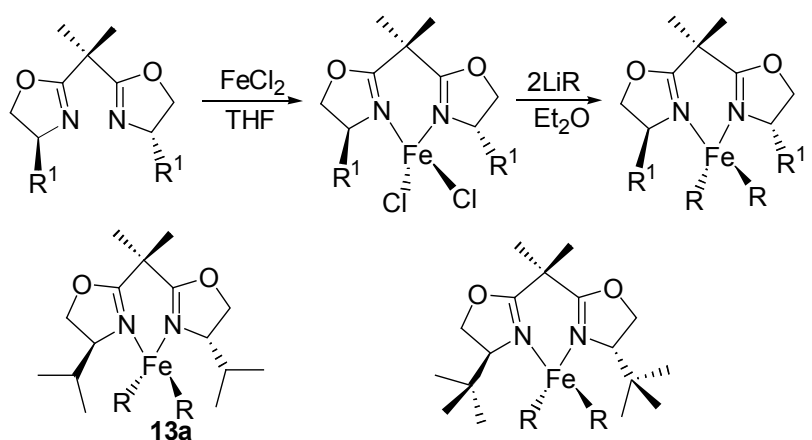

$(S, S)-(i-\mathrm{PrBox}) \mathrm{Fe}\left(\mathrm{CH}_{2} \mathrm{SiMe}_{3}\right)_{2}$ $\mathrm{R}=\mathrm{CH}_{2} \mathrm{SiMe}_{3} 99 \%, 9 \%$ ee $(\mathrm{S}, \mathrm{S})-(i-\mathrm{PrBOx}) \mathrm{Fe}\left(\mathrm{CH}_{2} \mathrm{CMe}_{3}\right)_{2}$ $\mathrm{R}=\mathrm{CH}_{2} \mathrm{CMe}_{3} \quad 99 \%, 17 \%$ ee

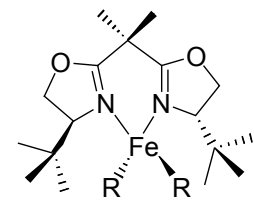

$13 b$

$(\mathrm{S}, \mathrm{S})-(t-\mathrm{BuBox}) \mathrm{Fe}\left(\mathrm{CH}_{2} \mathrm{SiMe}_{3}\right)_{2}$ $\mathrm{R}=\mathrm{CH}_{2} \mathrm{SiMe}_{3} \quad 99 \%, 2 \%$ ee

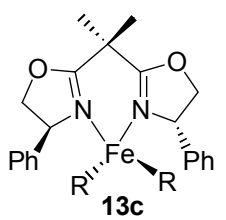

$(\mathrm{S}, \mathrm{S})-(\mathrm{PhBox}) \mathrm{Fe}\left(\mathrm{CH}_{2} \mathrm{CMe}_{3}\right)_{2}$ $\mathrm{R}=\mathrm{CH}_{2} \mathrm{CMe}_{3} 99 \%, 4 \%$ ee

图 $1213 \mathrm{a} \sim 13 \mathrm{c}$ 的结构

Figure 12 Structures of $13 a \sim 13 \mathbf{c}$

$\mathrm{H}_{2} \mathrm{SiPh}_{2}$ 和苯乙酮的硅氢加成反应. 室温下, 在手性膦 配体(一)-DIOP (15), (+)-Norphos (16), (一)-BPPFA (17) 存在下, 铜化合物 $\mathrm{CuO}_{2} \mathrm{CC}_{6} \mathrm{H}_{5}, \mathrm{CuOC}\left(\mathrm{CH}_{3}\right)_{3}$ 作为催化剂 (图 14)催化苯乙酮与二苯基硅烷反应, ee 值达到 10\% $40 \%$, 这是首次将铜配合物应用到催化硅氢加成反应 中, 虽然催化反应的光学选择性比较低, 但是具有非常 重要的参考价值.

2001 年, Lipshutz 课题组 ${ }^{[34]}$ 深入地研究了铜与配体 18, 19(图 15)催化酮不对称硅氢加成反应. 研究了 $\mathrm{CuH}$ 和手性 Roche 配体 $(R)$ 或 $(S)$-3,5-xyl-MeOBIPHEP (18)原 位制备的催化剂体系 ${ }^{[35,36]}$, 发现该催化体系能够很好地

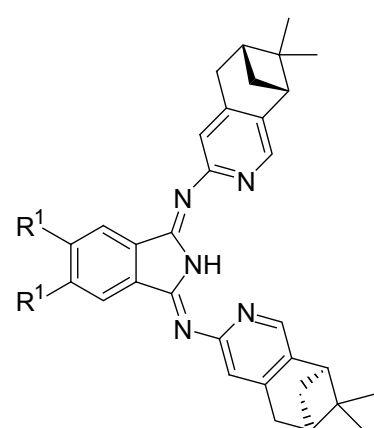

14a: $R^{1}=\mathrm{H}$

14b: $R^{1}=M e$

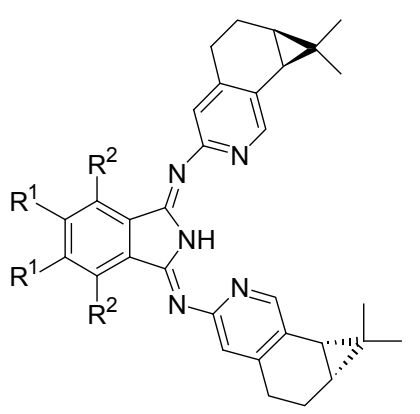

14f: $R^{1}, R^{2}=H$

14h: $R^{1}=M e, R^{2}=H$

14i: $R^{1}, R^{2}=P h$

图 $1314 \mathrm{a} \sim 14 \mathrm{i}$ 的结构

Figure 13 Structures of $14 a \sim 14 i$

催化芳香酮的不对称硅氢加成反应. 反应以苯乙酮为底 物, 过量的 PMHS(含氢聚硅氧烷)作为氢源, 催化剂用 量为底物的 $0.005 \mathrm{~mol} \%$, 反应的转化率达 $95 \%$ 以上, ee 值达 92\%. 同时, 催化剂可重复使用, 该催化体系具有 较大的应用潜力. Lipshutz 等 ${ }^{[37]}$ 还研究了温和条件下催 化杂环芳基酩的不对称硅氢加成反应. 研究表明，选择 配体 BIPHEP (18), SEGPHOS (19), 在 $-78 \sim-50{ }^{\circ} \mathrm{C}$ 的 


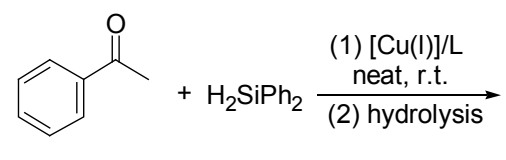

$[\mathrm{Cu}(\mathrm{I})] / \mathrm{L}=\mathrm{CuO}(t-\mathrm{Bu}) /(\mathrm{S}, \mathrm{S})-\mathrm{DIOP}$

$[\mathrm{Cu}(\mathrm{I})] / \mathrm{L}=\mathrm{Cu}\left(\mathrm{O}_{2} \mathrm{CC}_{6} \mathrm{H}_{5}\right) /(\mathrm{S}, S)$-Norphos

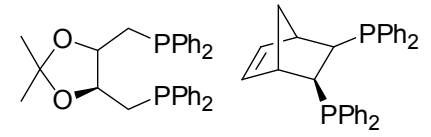

DIOP $15 \quad$ Norphos 16

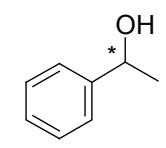

yield $100 \%, 20 \%$ ee yield $51 \%, 38.8 \%$ ee

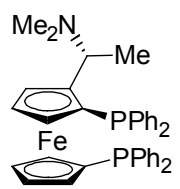

BPPFA 17
图 14 DIOP, Norphos, BPPFA 的结构

Figure 14 Structures of DIOP, Norphos, BPPFA, DPPF and BINAP

条件下, 以 PMHS 为氢源, $\mathrm{CuH}$ 具有最佳的催化活性, 反应的光学选择性高达 $90 \%$. Lipshutz 等 ${ }^{[38]}$ 还发现 $\mathrm{CuH}$ 与 DTBM-SEGPHOS (19a)配位的催化体系能很好地催 化具有生理活性的芳香酩的不对称硅氢加成反应，反应 的产率和立体选择性均高达 $86 \%$ 以上.<smiles>COc1cccc(OC)c1-c1c(OC)cccc1P(Br)(Br)(Br)Br</smiles>

BIPHEP 18<smiles>[R]c1cc(I)cc([R])c1[R]</smiles>

$\mathrm{R}=t-\mathrm{Bu}, \mathrm{R}^{\prime}=\mathrm{OMe} \quad \mathrm{DTBM}$

b: $R=M e, R^{\prime}=H \quad D M$
图 15 BIPHEP 和 SEGPHOS 的结构

Figure 15 Structures of BIPHEP and SEGPHOS

Dagorne 等 ${ }^{[39]}$ 研究表明 $(R)-\mathrm{BINAP} / \mathrm{CuCl} / \mathrm{NaO}-t-\mathrm{Bu}$ 体系能够很好地催化芳基烷基酮的不对称硅氢加成反 应. 实验中选用了众多硅烷作为氢源, 如二苯基硅烷、 甲基苯基硅烷、苯基叔丁基硅烷、1-菜基苯基硅烷、邻 甲苯基苯基硅烷, 结果表明以甲基苯基硅烷作为氢源的 立体选择性最高. Yun 等 ${ }^{[40]}$ 研究了在空气和水中均能稳 定存在的铜(II)盐用于催化芳香酮的硅氢加成反应. 以 水合醋酸铜(II)或醋酸铜(II)与 BINAP 配位作为催化剂, 可以还原酮得到醇. 利用 $\mathrm{Cu}(\mathrm{OAc})_{2} \cdot \mathrm{H}_{2} \mathrm{O}$ 替代 $\mathrm{CuCl} /$ $\mathrm{NaO}-t-\mathrm{Bu}$ 体系原位产生 $\mathrm{CuH}$ 来催化酩的不对称硅氢加 成反应, 实验操作简单、方便, 化学和光学产率达 $80 \%$ 以上.

2010 年, Junge 等 ${ }^{[41]}$ 用醋酸铜或醋酸铜水合物以及 膦配体 20, 如图 16, 以 1 equiv. 苯基含氢硅烷为氢源, 在一 $20{ }^{\circ} \mathrm{C}$ 下, 以甲苯为溶剂, 催化不同的酮底物, 最终 得到手性二级醇. 以对氟苯乙酤为底物时产率最高, 而 且光学选择性也有 $80 \%$; 以对甲氧基苯乙酮为底物时,
光学选择性最高达 $91 \%$, 但产率却只有 57\%.

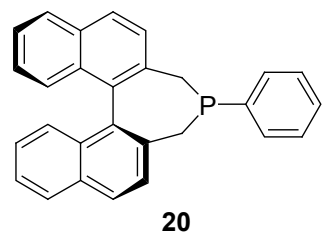

图 16 膦配体

Figure 16 Phosphine ligands

Riant 等 ${ }^{[42]}$ 指出 $\mathrm{CuF}_{2}$ (1 $\mathrm{mol} \%$ )- 手性双膦配体 BINAP 催化各种酩的不对称硅氢加成反应, 获得了 $78 \% \sim 92 \%$ 的 $e e$ 值. 实验表明，氟能在催化循环的第一 步中通过活化 $\mathrm{Si}-\mathrm{H}$ 键产生 $\mathrm{Cu}(\mathrm{I})-\mathrm{H}$. 氧气能加速反应 的进行, $\mathrm{CuF}_{2}$ /二齿膦配体/苯基硅烷催化体系在空气中 更稳定，反应活性更高，反应时间更短. $\mathrm{Wu}$ 等 ${ }^{[43,44]}$ 利用 $\mathrm{CuF}_{2}, \mathrm{Cu}(\mathrm{acac})_{2}$ 与手性二吡啶磷配体 $S-21 \mathbf{a}, S-21 \mathbf{b}$ 催化 潜手性酮合成手性醇，反应条件温和，转化率和光学选 择性都比较高, 光学选择性最高可以达到 $99 \%$, 如图 17.

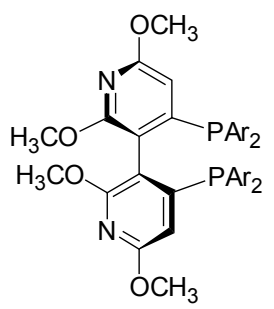

$\mathrm{Ar}=\mathrm{C}_{6} \mathrm{H}_{5}:(S)-\mathbf{2 1 a},(\mathrm{S})-\mathrm{P}-\mathrm{Phos}$

$\mathrm{Ar}=3,5-\left(\mathrm{CH}_{3}\right)_{2} \mathrm{C}_{6} \mathrm{H}_{3}:(\mathrm{S})-\mathbf{2 1 b},(\mathrm{S})-\mathrm{Xyl}-\mathrm{P}-\mathrm{Phos}$

$$
\stackrel{\begin{array}{l}
\text { 1) } \mathrm{CuF}_{2}(1 \mathrm{~mol} \%) \text {, ligand }(1 \mathrm{~mol} \%) \\
\mathrm{PMHS}_{\mathrm{R}}\left(5 \text { equiv.), toluene, }-20{ }^{\circ} \mathrm{C}\right.
\end{array}}{\text { 2) aqueous } \mathrm{NaOH}}
$$

图 $17 \mathrm{Cu}(\mathrm{II})$ /手性二吡啶膦配体化合物催化酮硅氢加成反应 Figure $17 \mathrm{Cu}(\mathrm{II}) /$ dipyridylphosphine ligands catalyzed asymmetric hydrosilylation of ketones

最近 Dedieu 等 ${ }^{[45]}$ 通过实验和理论计算提出了 $\mathrm{Cu}(\mathrm{I})$ 与手性膦配体催化潜手性酮的不对称硅氢加成反应机 理, 如图 18 所示. 首先 $\mathrm{Cu}(\mathrm{I})$ 的手性膦络合物与含氢硅 烷生成铜的氢化物种 $\left(\mathrm{L}^{*}-\mathrm{Cu}-\mathrm{H}\right)$, 接着酩插入到铜的 氢化物中，形成铜的醇盐中间体，这是不可逆的一步反 应，决定了反应的不对映选择性，接着铜的醇盐与氢硅 烷反应，经过中间体，生成稳定的硅醚，同时释放出铜 的氢化物种, 这一步决定了反应的速率, 生成稳定硅醚 是反应的动力.

现在，氮杂环卡宾铜配合物作为催化剂应用于催化 酮的不对称硅氢加成反应受到越来越多的科研工作者 的关注. Buchmeiser 等 ${ }^{[46]}$ 以 1,3-二烃基-3,4,5,6-四氢嘧啶 鎓盐为配体，合成具有手性结构的单卡宾配位的 $\mathrm{Cu}$ 卡 


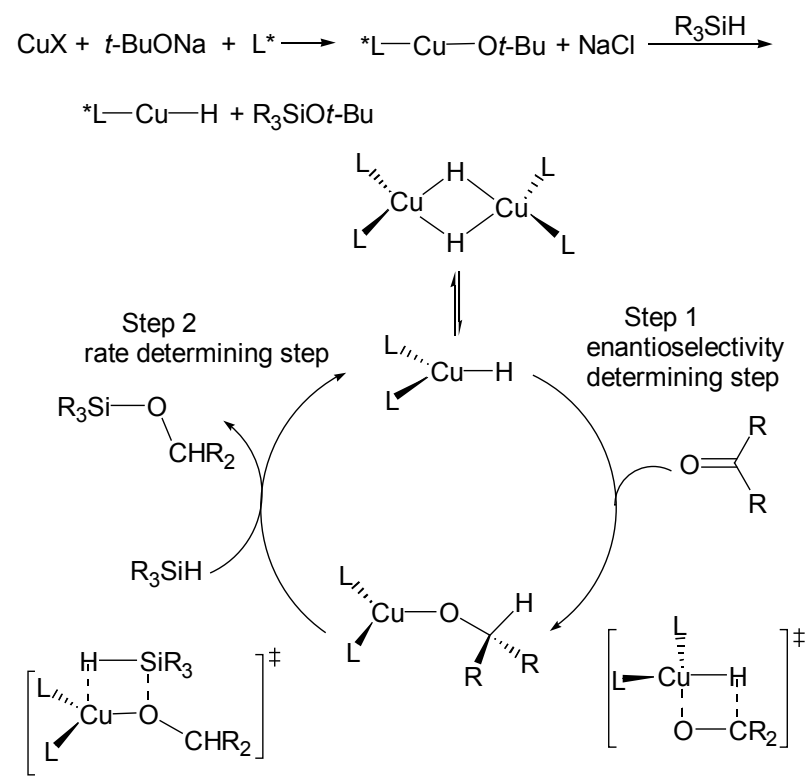

图 $18 \mathrm{Cu}(\mathrm{I})$ 催化潜手性酮的不对称硅氢加成反应机理

Figure 18 Mechanism of asymmetric hydrosilylation of ketones catalyzed with $\mathrm{Cu}(\mathrm{I})$

宾络合物 22a, 22b 和双卡宾配位的 $\mathrm{Cu}$ 卡宾络合物 22c, 如图 19, 研究了 22b 和 22c 催化不同芳基酮的硅氢加成 反应，发现双卡宾配位的 $\mathrm{Cu}$ 卡宾络合物 22c 的催化活 性高于单卡宾配位的 $\mathrm{Cu}$ 卡宾络合物 $\mathbf{2 2 a}, \mathbf{2 2 b}$.
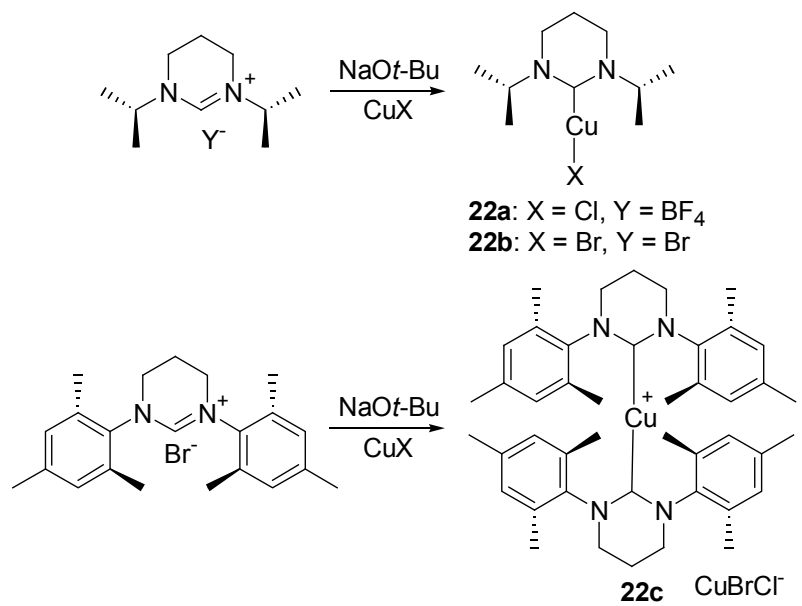

图 $19 \mathrm{Cu}$ 卡宾络合物 22

Figure 19 Copper carbene complex 22

2011 年, Gawley 等 ${ }^{[47]}$ 用具有 $C_{2}$ 轴对称结构的氮杂 环卡宾铜配合物 23 催化潜手性的酮的不对称硅氢加成 反应, 其催化性能非常突出, 在室温下, $0.5 \sim 2 \mathrm{~h}$ 内不仅 能高产率高立体选择性的催化芳基酮, 反应产率 $\geqslant$ $85 \%$, 光学选择性 $\geqslant 94 \%$, 而且能够高效的催化具有极 具挑战性的 2-丁酮和 2-戊酮的硅氢加成, 产率分别为 $62 \%$ 和 $77 \%$, 光学选择性分别可以达到 $96 \%$ 和 $90 \%$, 如 图 20 .

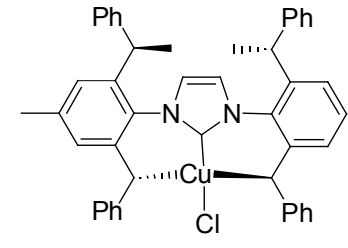

$(R, R, R, R)$-CuPhEt

23

图 20 手性氮杂环卡宾铜配合物

Figure 20 Chiral copper-N-heterocyclic carbene complex

相对于 $\mathrm{Zn}, \mathrm{Fe}$ 等其它非贵金属催化体系而言, $\mathrm{Cu}$ 催 化体系研究得比较成熟, 配体呈现出多样性, 其工业应 用前景广阔 ${ }^{[48]}$.

\section{4 潜手性酮不对称硅氢加成反应中的钛催化剂}

1994 年, Buchwald 等 ${ }^{[49]}$ 首次报道了手性二茂钛催 化剂能够催化芳香酮与 PMHS 的不对称硅氢加成反应, 如图 21. 底物为苯乙酮时, ee 值为 $97 \%$, 但该催化剂只 对具有共轭体系的酮具有很好的不对映选择性，并且催 化量达到 $4.5 \% \sim 10 \%$, 反应时间也长达 $1 \sim 4.5 \mathrm{~d}$. 因此, 1999 年, Buchwald 课题组 ${ }^{[50]}$ 优化了手性二茂钛催化反 应条件, 如图 22 所示. 通过滴加甲醇, 反应速率大大提 高, 反应时间缩短到 $3 \sim 10 \mathrm{~h}$, 并且催化量减少到 $0.5 \% \sim 2 \%$ ，部分芳基酮的光学选择性可达到 $99 \%$ 并且 对饱和酩的不对映选择性也明显提高. 这使得该催化体 系有了一定的工业应用价值.

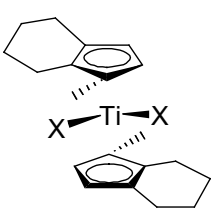

24

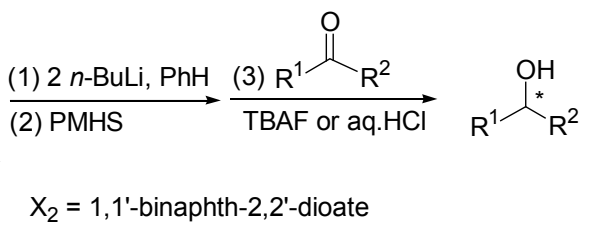

图 21 二茂钛 24 催化不对称硅氢加成反应

Figure 21 Titanocene $\mathbf{2 4}$ catalyzed asymmetric hydrosilylation of ketones

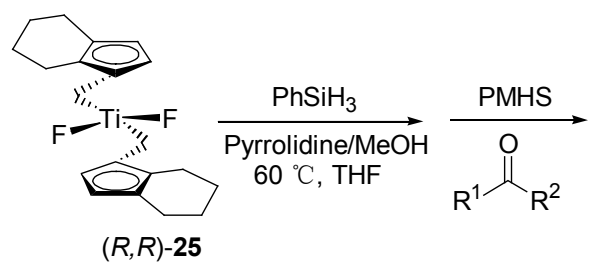

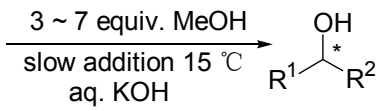

图 22 二茂钛 25 催化不对称硅氢加成反应

Figure 22 Titanocene $\mathbf{2 5}$ catalyzed asymmetric hydrosilylation of ketones

同时, Buchwald 等 ${ }^{[50]}$ 提出了手性二茂钛催化酮的反 
应机理, 如图 23 所示, 合理解释了滴加一级醇反应速率 和不对映选择性者提高的原因. 所提出的机理表明，决 定反应速率和反应的不对映选择性是两条不同反应路 径, 反应速率是由 $\mathrm{Ti}-\mathrm{O}$ 键和 $\mathrm{Si}-\mathrm{H}$ 键之间 $\sigma$ 键的交换 决定的, 通过滴加一级醇能提高反应速率是由于在决定 反应速率步骤中减少了 $\mathrm{OR}$ 基团的位阻 (OR' 比 $O C H R^{1} R^{2}$ 位阻小得多), 因此反应速率提高. 而反应不 对称选择性的提高是由于醇的滴加相加快了有选择性 的不对称硅氢反应路径的反应速率，从而提高反应的不 对称选择性.

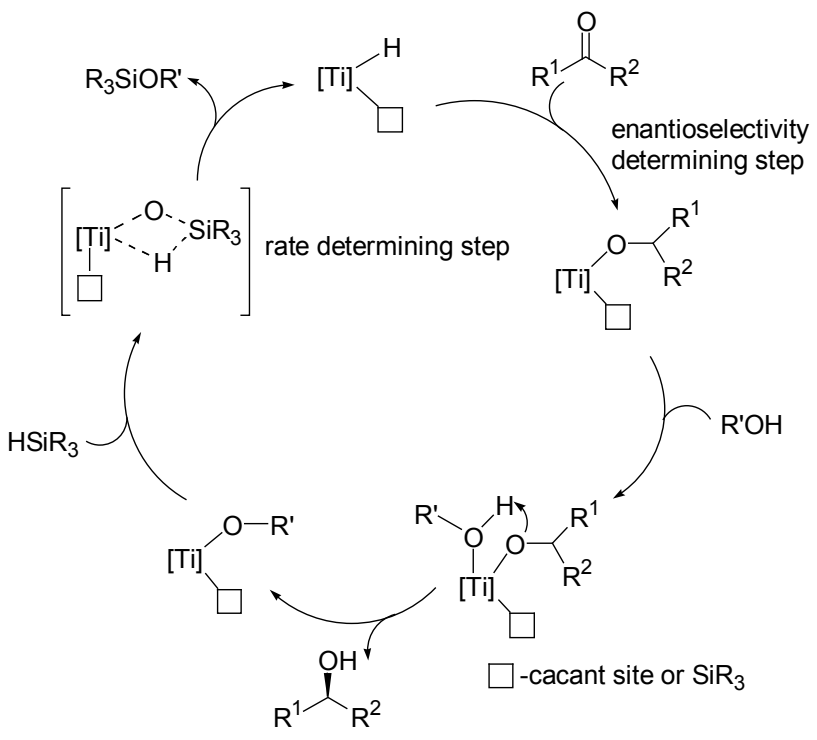

图 23 手性二茂钛催化酮的不对称硅氢加成反应机理

Figure 23 Mechanism of hydrosilylation of ketones catalyzed with titanocene

Beagley 课题组 ${ }^{[51,52]}$ 利用新型手性二茂钛催剂 $(R)-$ 或 $(S)-\left[\mathrm{TiCl}_{2}\left(\eta^{5}: \eta^{5}-\mathrm{C}_{5} \mathrm{Me}_{4} \mathrm{SiMe}_{2} \mathrm{C}_{5} \mathrm{H}_{3} \mathrm{R}^{*}\right)\right]\left(\mathrm{R}^{*}=\right.$ Menthyl, Neomenthyl), 如图 24 所示, 室温下, 在甲苯溶液中以 $\mathrm{PhSiH}_{3}$ 为还原剂, 催化酮的不对称硅氢加成反应, 光学 选择性最高达到 $82 \%$ ，同时转化率达到 $100 \%$.

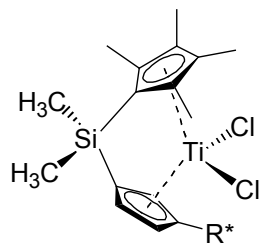

$(R)$

26a $R^{*}=$ menthyl

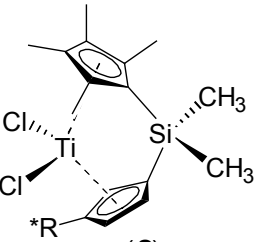

(S) 26b $R^{*}=$ neomenthyl

图 24 钛催化剂 26

Figure 24 Titanium catalyst 26

钛催化酮的不对称硅氢加成不只是局限于二茂钛 催化体系, Nakai 等 ${ }^{[53]}$ 合成了新型的二齿配体的钛催化
剂 27, 如图 25 所示, 用于催化苯乙酮与三乙氧基硅烷 的不对称硅氢加成反应，水解得到相应的醇，ee 值为 $55 \%$.

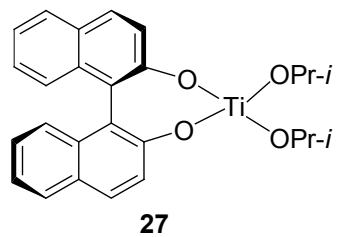

图 25 钛催化剂 27

Figure 25 Titanium catalyst 27

Cozzi 等 ${ }^{[54,55]}$ 合成了的盒子状配体 $\mathbf{2 8}$, 与 $\mathrm{TiF}_{4}$ 原位 催化芳基酮的不对称硅氢加成反应，光学选择性最高可 达到 $85 \%$.<smiles>c1ccc([C@@H]2N=C(CC3=N[C@H](c4ccccc4)[C@H](c4ccccc4)O3)O[C@H]2c2ccccc2)cc1</smiles>

图 26 配体 $\mathbf{2 8}$ 的结构

Figure 26 Structures of $\mathbf{2 8}$

\section{5 结论与展望}

不对称硅氢加成反应自 20 世纪 80 年代以来发展迅 速，在基础研究和开发手性配体方面都取得了很大的成 功. 非贵金属催化剂催化不对称硅氢加成的应用在不对 称催化研究中占有非常重要的地位，尽管已经取得了一 定成绩，但这一领域仍然有许多尚未解决的问题，合成 新颖的、简单的、可操作性强的、具有高立体选择性手 性配体仍然是今后研究的重要方向.

\section{References}

[1] Ojima, I. In Chemistry of Organic Silicon Compounds, Vol. 2, Eds.: Patai, S.; Rappoport, Z., Wiley, New York, 1998, p. 1687.

[2] (a) Ojima, I.; Li, Z.; Zhu, J. In Recent Advances in the Hydrosilylation Reaction: Chemistry of Organic Silicon Compounds, Vol. 2, Eds.: Rappoport, Z.; Apeloig, Y., Wiley, New York, 1998.

(b) Yamamoto, K.; Uramoto, Y.; Kumada, M. J. Organomet. Chem. 1971, 31, C9.

[3] (a) Brunner, H.; Becker, R.; Riepl, G. Organometallics 1984, 3, 1354.

(b) Nishiyama, H.; Yamaguchi, S.; Kondo, M.; Itoh, K. J. Org. Chem. 1992, 57, 4306.

[4] Li, J. Y.; Peng, J. J.; Bai, Y.; Hu, Y. Q.; Zhang, G. D.; Lai, G. Q. Chin. J. Org. Chem. 2009, 29, 1938 (in Chinese). (厉嘉云, 彭家建, 白赢, 胡应乾, 张国栋, 来国桥, 有机化学, 2009, 29, 1938.)

[5] (a) Lin, G. Q.; Chen, Y. Q.; Chen, X. Z.; Li, Y. M. Chiral SynthesisAsymmetric Reactions and Applications, Science Press, Beijing, 2000, p. 262 (in Chinese). 
(林国强, 陈耀全, 陈新滋, 李月明著, 手性合成一一不对称反 应及其应用, 科学出版社, 北京, 2000, p. 262.)

(b) Wishka, D. G.; Graber, D. R.; Seest, E. P.; Dolak, F. H.; Watt, W.; Morris, J. J. Org. Chem. 1998, 63, 7851.

[6] (a) Matsumura, K.; Hashiguchi, S.; Ikariya, T.; Noyori, R. J. Am. Chem. Soc. 1997, 119, 8738.

(b) Haack, K. J.; Hashiguchi, S.; Fuji, A.; Ikariya, T.; Noyori, R. Angew. Chem., Int. Ed. 1997, 36, 285.

[7] (a) Fiaud, J. C.; Kagan, H. B. Bull. Soc. Chim. Fr. 1969, 2742. (b) Corey, E. J.; Bakshi, R. K.; Shibata, S. J. Am. Chem. Soc. 1987, $109,5551$.

(c) Corey, E. J.; Bakshi, R. K.; Shibata, S.; Chen, C. P.; Singh, V. K. J. Am. Chem. Soc. 1987, 109, 7925.

(d) Cai, Z. Y.; Zhou, W. C. Chin. J. Org. Chem. 2008, 28, 937 (in Chinese).

(蔡正艳, 周伟澄, 有机化学, 2008, 28, 937.)

[8] Dumont, W.; Poulin, J. C.; Phat, T. D.; Kagan, H. B. J. Am. Chem. Soc. 1973, 95, 8295.

[9] Langlois, N.; Dang, T. P.; Kagan, H. B. Tetrahedron Lett. 1973, 14, 4865.

[10] Brunner, H.; Becker, R.; Riepl, G. Organometallics 1984, 3, 1354.

[11] Nishibayashi, Y.; Segawa, K.; Ohe, K.; Uemura, S. Organometallics 1995, 14, 5486.

[12] Ojima, I.; Kogure, T.; Nagai, Y. Tetrahedron Lett. 1974, 15, 1889.

[13] Zhu, G.; Terry, M.; Zhang, X. J. Organomet. Chem. 1997, 547, 97.

[14] Enders, D.; Gielen, H.; Breuer, K. Tetrahedron: Asymmetry 1997, 8, 3571.

[15] Mimoun, H.; de Saint Laumer, J. Y.; Giannini, L.; Scopelliti, R.; Floriani, C. J. Am. Chem. Soc. 1999, 121, 6158.

[16] Bette, V.; Mortreux, A.; Lehmann, C. W.; Carpentier, J. F. Chem. Commun. 2003, 332.

[17] Bette, V.; Mortreux, A.; Ferioli, F.; Martelli, G.; Savoia, D.; Carpentier, J. F. Eur. J. Org. Chem. 2004, 14, 3040.

[18] Bette, V.; Mortreux, A.; Savoia, D.; Carpentier, J.-F. Tetrahedron 2004, 60, 1889.

[19] Bette, V.; Mortreux, A.; Savoia, D.; Carpentier, J.-F. Adv. Synth. Catal. 2005, 16, 2837.

[20] Ushio, H.; Mikami, K. Tetrahedron Lett. 2005, 46, 2903.

[21] Inagaki, T.; Yamada, Y.; Phong, L. T.; Furuta, A.; Ito, J. I.; Nishiyama, H. Synlett 2009, 0253.

[22] Gajewy, J.; Kwit, M.; Gawrońskia, J. Adv. Synth. Catal. 2009, 351, 1055.

[23] Junge, K.; Möller, K.; Wendt, B.; Das, S.; Gördes, D.; Thurow, K.; Beller, M. Chem. Asian J. 2012, 7, 314.

[24] Liu, S.; Peng, J. J.; Yang, H.; Li, J. Y.; Bai, Y.; Lai, G. Q. Tetrahedron 2012, 68, 1371.

[25] Brunner, H.; Eder, R.; Hammer, B.; Klement, U. J. Organomet. Chem. 1990, 394, 555.

[26] Nishiyama, H.; Furuta, A. Chem. Commun. 2007, 760.

[27] Inagaki, T.; Ito, A.; Ito, J.-I.; Nishiyama, H.; Angew. Chem., Int. Ed. 2010, $49,9384$.

[28] Inagaki, T.; Phong, L. T.; Furuta, A.; Ito, J.-I.; Nishiyama, H. Chem.
Eur. J. 2010, 16, 3090

[29] Hosokawa, S.; Ito, J.; Nishiyama, H. Organometallics 2010, 29, 5773.

[30] Shaikh, N. S.; Enthaler, S.; Junge, K.; Beller, M. Angew. Chem., Int. Ed. 2008, 47, 2497.

[31] Tondreau, A. M.; Darmon, J. M.; Wile, B. M.; Floyd, S. K.; Lobkovsky, E.; Chirik, P. J. Organometallics 2009, 28, 3928.

[32] Langlotz, B. K.; Wadepohl, H.; Gade, L. H. Angew. Chem., Int. Ed. 2008, 47, 4670

[33] Brunner, H.; Becker R.; Riepl, G. A. Organometallics 1984, 3, 1354.

[34] Lipshutz, B. H.; Chrisman, W.; Noson, K. J. Organomet. Chem. 2001, 624, 367.

[35] Lipshutz, B. H.; Noson, K.; Chrisman, W. T. J. Am. Chem. Soc. 2001, 123, 12917.

[36] Yun, J.; Buchwald, S. L. Org. Lett. 2001, 3, 1129.

[37] Lipshutz, B. H.; Lower, A.; Noson, K. Org. Lett. 2002, 4, 4045.

[38] Lipshutz, B. H.; Lower, A.; Kucejko, R. J.; Noson, K. Org. Lett. 2006, 8, 2969.

[39] Issenhuth, J. T.; Dagorne, S.; Bellemin-Laponnaza, S. Adv. Synth. Catal. 2006, 348, 1991.

[40] Lee, D. W.; Yun, J. Tetrahedron Lett. 2004, 45, 5415.

[41] Junge, K.; Wendt, B.; Addis, D.; Zhou, S.; Das, S.; Beller, M. Chem. Eur. J. 2010, 16, 68.

[42] Sirol, S.; Courmarcel, J.; Mostefai, N.; Riant, O. Org. Lett. 2001, 3, 4111.

[43] Zhang, X. C.; Wu, Y.; Yu, F.; Wu, F. F.; Wu, J.; Chand, A. S. C. Chem. Eur. J. 2009, 15, 5888.

[44] Zhang, X. C.; Wu, F. F.; Li, S. J.; Zhou, J. N.; Wu, J.; Li, N.; Fang, W. J.; Lam, K. H.; Chand, A. S. C. Adv. Synth. Catal. 2011, 353, 1457.

[45] Issenhuth, J.-T.; Notter, F.-P.; Dagorne, S.; Dedieu, A.; BelleminLaponnaz, S. Eur. J. Inorg. Chem. 2010, 4, 529.

[46] Bantu, B.; Wang, D.; Wurst, K.; Buchmeiser, M. R. Tetrahedron 2005, 61, 12145 .

[47] Albright, A. E.; Gawley, R. J. Am. Chem. Soc. 2011, 133, 19680.

[48] Chen, L.-Z.; Peng, J. J.; Li, J. Y.; Bai, Y.; Qiu, H.-Y.; Lai, G. Q. Chin. J. Org. Chem. 2008, 28, 761 (in Chinese). (陈玲珍，彭家建，厉嘉云，白赢，邱化玉，来国桥，有机化学， 2008, 28, 761.)

[49] Carter, M. B.; Schiøtt, B.; Gutiérrez, A.; Buchwald, S. L. J. Am. Chem. Soc. 1994, 116, 11667.

[50] Yun, J.; Buchwald, S. L. J. Am. Chem. Soc. 1999, 121, 5640.

[51] Beagley, P.; Davies, P. J.; Blacker, A. J.; White, C. Organometallics 2002, 21, 5852.

[52] Beagley, P.; Davies, P. J.; Adams, H.; White, C. Can. J. Chem. 2001, 79, 731.

[53] Imma, H.; Nori, M.; Nakai, T. Synlett 1996, 1229.

[54] Bandini, M.; Cozzi, P. G.; Negro, L.; Umani-Ronchi, A. Chem. Commun. 1999, 39.

[55] Bandini, M.; Bernardi, F.; Bottoni, A.; Cozzi, P. G.; Miscione, G. P.; Umani-Ronchi, A. Eur. J. Org. Chem. 2003, 15, 2972. 